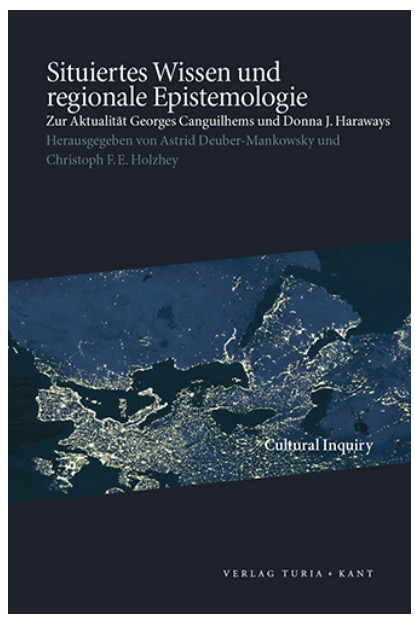

Situiertes Wissen und regionale Epistemologie: Zur Aktualität Georges Canguilhems und Donna J. Haraways, ed. by Astrid Deuber-Mankowsky and Christoph F. E. Holzhey, Cultural Inquiry, 7 (Wien: Turia + Kant, 2013), pp. 161-73

\section{MONIQUE DAVID-MÉNARD \\ Die Begrenzung der Wissensfelder bei Kant, Canguilhem und Foucault}

CITE AS:

\begin{abstract}
Monique David-Ménard, »Die Begrenzung der Wissensfelder bei Kant, Canguilhem und Foucault $\ll$, in Situiertes Wissen und regionale Epistemologie: Zur Aktualität Georges Canguilhems und Donna J. Haraways, hg. v. Astrid Deuber-Mankowsky und Christoph F. E. Holzhey, Cultural Inquiry, 7 (Wien: Turia + Kant, 2013), S. 161-73 <https: //doi.org/10.37050/ci-07_08>
\end{abstract}

RIGHTS STATEMENT:

(C) by the author(s)

This version is licensed under a Creative Commons AttributionShareAlike 4.0 International License. 


\section{DIE BEGRENZUNG DER WISSENSFELDER BEI KANT, CANGUILHEM UND FOUCAULT}

Monique David-Ménard

Mit ihrer Forderung nach einer Situierung des Wissens kritisiert Donna J. Haraway den Anspruch der Wissenschaften, insbesondere der Biologie, ubiquitäre Erkenntnisse zu liefern, deren universeller Wert eben von dieser Ubiquität abhängt. Wenn ich im Folgenden deutlich machen werde, was die drei im Titel dieses Beitrags genannten Autoren Kant, Canguilhem und Foucault miteinander verbindet, möchte ich damit für eine Regionalität des Wissens eintreten, die sich mit Haraways Position deckt und zugleich von ihr unterscheidet.

Ich habe in meinem Buch über Kants Auseinandersetzung mit Swedenborg La Folie dans la raison pure und später in Constructions de l'universel (Konstruktionen des Allgemeinen) zu zeigen versucht, dass die Universalität des Denkens, die für bestimmte Aussagen kennzeichnend ist, nicht so sehr illusorisch als vielmehr »uninteressant " ist. ${ }^{1}$ Ich bin dabei einer kritischen Analyse gefolgt, die sich methodisch auf die Psychoanalyse stützt, ohne die philosophischen Texte auf eine »Triebstruktur « zu reduzieren, durch die sie entwertet würden. »Uninteressant " scheint mir die Universalität des Denkens in dem Sinne, da, um mit Deleuze zu sprechen, die Frage, ob eine Philosophie wahr sei, keine interessante Frage ist. Ich will hiermit sagen, dass die Universalität des Denkens in der Philosophie Effekt einer Abspaltung [détachement] ist, die in philosophischen Texten zum Tragen kommt. Diesen Vorgang der Abspaltung oder Ablösung genauer verorten zu können - »Ablösung « hier in einem aktiven Wortsinn, gleichsam als roter Faden, verstanden, der nicht nur für die Entstehung, sondern auch für die Systematizität eines Werks entscheidend ist - würde der Ubiquität, die von bestimmten Denkweisen erzeugt wird, den Status eines Effekts und nicht eines Prinzips verleihen. Dabei bleibt gleichfalls die Frage offen, ob die gebieterische Notwendigkeit, sich durch die Ausübung des Denkens von sich selbst zu trennen, allein schon eine relative Universalität der Aussagen oder Schreibweisen zur Folge hat, wenn es sich um solche einer Wissenschaft handelt. ${ }^{2}$ 
Mit Blick auf eine regionale Epistemologie möchte ich an dieser Stelle ein anderes Argument entwickeln: In einer Befragung der Grenzen des Wissens wird es darum gehen, genau zu ermitteln, wie eine Erfindung des Denkens einen ihr vorgängigen Kontext voraussetzt, von dem sich neue Begriffe loslösen. »Sich ablösen« bedeutet also keineswegs, den anthropologischen oder triebhaften Boden zu verkennen, von dem es sich durch das Denken frei zu machen gilt. Es handelt sich eher darum, eine dem Wissen vorausliegende Illusion kritisieren zu können. Das mit dieser Kritik gleichzeitig produzierte Wissen ist also notwendigerweise durch die Verlaufsbahn eben dieser Kritik situiert bzw. lokalisiert. Diese Problematik einer Kritik an vorausgehenden Illusionen ist allerdings weder mein Anliegen, noch mein persönliches Spezialgebiet. Seit Kant, Comte, Bachelard, Foucault und Althusser gibt es eine ganze philosophische Tradition, die die Problematik der epistemologischen Brüche genutzt (und sicherlich mitunter auch ausgenutzt) hat. Doch dass es in Frankreich in den Jahren zwischen 1960 und 1980 eine, übrigens wieder schnell in Vergessenheit geratene Mode der epistemologischen Hindernisse und Brüche gab, entscheidet noch nicht über die Relevanz dieser Begriffe, wenn es darum geht zu ermitteln, wie man in bestimmten Kontexten "anders denken $\aleph^{3}$ kann.

Es stehen sich hier zwei Traditionen gegenüber, die sich mit den Namen von Spinoza und Kant benennen lassen: Für Spinoza muss die Vernunft nicht zeigen, wie sie aus der Illusion hervorgegangen ist, da sich die Begriffe Irrtum und Illusion immer erst nachträglich ergeben. An erster Stelle steht die Tatsache, dass wir, wie aus dem ersten Satz der Ethik folgt, »eine wahre Idee« haben. Diese »wahre Idee« entspringt nicht der Kritik der Ideen, die der ersten oder zweiten Erkenntnisgattung angehören. Dementsprechend ist das Wissen nicht situiert. Wenn wir eine wahre Idee haben, sind wir nicht nur im Absoluten, sondern wir sind das Absolute, ein Teil des göttlichen Verstands. Im Gegensatz dazu werden für Kant bestimmte Gedanken Illusionen oder dem Wahn abgewonnen - sei es der »Wahn« eines »Geistersehers « oder sei es die der Vernunft innewohnende Wahnvorstellung, deren Mechanismen die Kritik in der »transzendentalen Dialektik « auseinander nimmt. Der Umstand nun, dass eine gedankliche Schöpfung aus einer Illusion gewonnen wird, weist dem Denken einen unsicheren Standort zu; denn ob sich Denken und Illusion voneinander trennen lassen, ist hier ungewiss. 
Welche Beziehung besteht also zwischen der Problematik der Grenzen des Wissens und dem In-Rechnung-Stellen der Illusionen, von denen sich das Wissen abgrenzt? Auf diese Weise formuliert, gilt diese Frage in gleicher Weise für Kant, für Canguilhem und auch für Foucault. An dieser Stelle geht es mir jedoch darum, die Unterschiede zwischen ihren Positionen zu verdeutlichen. Entscheidend dabei ist, dass die Hervorbringung eines neuen Wissens einen Kontext voraussetzt, aus dem es sich löst. Denn es sind die Modalitäten dieser Ablösung, die verhindern, dass man einen übergeordneten Standpunkt einnehmen kann. Die Erfindungskraft des Denkens hängt von dem skeptischen Moment ab, das es entfaltet bzw. austrägt. Mit Blick auf das »skeptische Moment ", in dem die Kreation von Begriffen sich entfaltet, möchte ich die unterschiedlichen Spielarten dieses Moments bei Kant, Canguilhem und Foucault durchgehen, das die Begrenzung eines Wissensfelds oder vielmehr zweier sich ausdifferenzierender Wissensfelder impliziert. Die Begriffserfindung findet im Denken der Differenz dieser zwei Felder statt. Aus diesem Grund habe ich oben auch die Gleichzeitigkeit der Frage nach der Grenze des Wissens und dessen Ausgang aus einer Illusion hervorgehoben. Abschließend möchte ich die vorgestellte Konzeption des »skeptischen Moments « Hegels Analyse des Skeptizismus gegenüberstellen, der zufolge der Skeptizismus fälschlicherweise einem Gegebenen verpflichtet bleibt, das er auf stets gleiche Weise anficht. Denn es ist gerade die Tatsache, dass die Kritik eines vorausgesetzten Wissens immer auf singuläre Weise geschieht, welche eine Erfindung von neuen Begriffen allererst ermöglicht.

\section{KANT: DIE BEGRENZUNG DES FELDS DER PHÄNOMENE SETZT EIN LEERES "JENSEITS " VORAUS}

Ein paar Worte zur Erinnerung über die Problematik der Grenzen bei Kant, der die Begrenztheit des Wissensfelds in einem positiven Sinne auffasst: Wissen gibt es allein unter der Bedingung, dass dieses seinen Gegenstand zu konstituieren weiß. Und der Gegenstand ist hier weniger das Korrelat eines Subjekts als vielmehr die durch eine Grenzsetzung bewirkte Modifikation eines Objekts. Dieses wird dem illusionären Denken insofern abgerungen, als es der Synthese (die den Gegenstand in der Wahrnehmung erfasst und die von sich aus zum Unendlichen strebt) gelingt, sich selbst zu begrenzen. Die Gegenstände sind der Idee einer Welt abgewonnen, zu der sich kein Korrelat in der Realität finden lässt. 
Wie Françoise Balibar in ihrem Beitrag darlegt, verliert der Begriff der Objektivität in gegenwärtigen Wissenschaften an Bedeutung. ${ }^{4}$ Und Kant gilt als der Philosoph der Objektivität, der Gegenständlichkeit, schlechthin! Doch ist er mehr als das: »Gegenständlich « ist das Feld des Wissens, das durch die Transformation einer problematischen Vorstellung erzeugt wird, die auf das Unendliche ausgreift - doch dies eben nur im »reinen Denken« und ohne mögliches reales Korrelat. Dieses Korrelat kann laut Kant »nicht unter die Möglichkeiten gezählt werden «, auch wenn es formallogisch nicht widersprüchlich ist.

Zweitens ist daran zu erinnern, dass es bei Kant mindestens zwei Begriffe für Begrenzung gibt: die Schranke und die Grenze. So erlauben die problematischen Vernunftideen nur insofern die Gültigkeit eines Wissen, das ein Reales ins Spiel bringt, als es ihnen gelingt, die Synthese einzuschränken, durch die sie sich selbst unter den Bedingungen der Anschauung herausbilden. Selbst diese Beschränkung ist bei Kant positiv. Auch wenn sich die Begrenzungen als relativ unwirksame Grenzsteine zu erweisen scheinen, so macht dennoch die Setzung dieser Grenzen die Grenzsteine zu einem Produkt der aktiven Begrenzung des Feldes der Vernunft durch diese selbst. Dies ist es, was Kant mit Grenze und Begrenzung bezeichnet.

Drittens schließlich - und dies ist für Kants Zugehörigkeit zum achtzehnten Jahrhundert bezeichnend und unterscheidet ihn von Canguilhem und Foucault - erfordert die Idee der Begrenzung der Wissensfelder die Etablierung eines Gegensatzes von noumenon und phaenomenon. Die Endlichkeit des Felds der Phänomene ist allein dadurch gesichert, dass sich ein Objekt denken lässt, das rein intellektueller Natur ist und dessen empirischer Inhalt durch die Kritik als leer bzw. unter dem Gesichtspunkt der Erkenntnis als ein »Nichts « festgelegt wird. Das Ding an sich ist leer, doch es behält nach Kant einen Platz »jenseits « der Phänomene. Ohne die kritische Aktivität des Denkens, die das Jenseits für leer erklärt, hielte sich das Phänomen für absolut, wodurch seine Begrenzung nicht mehr gesichert wäre.

Man könnte noch weiter auf dieses subtile Geflecht der drei Aspekte der Begrenzung des Felds der Phänomene bei Kant eingehen. Insbesondere in der "Amphibolie der Reflexionsbegriffe " wird in einer Auseinandersetzung mit Leibniz dieser Zusammenhang weiter entfaltet. Leibniz schreibt seine Theorie der »kleinen Wahrnehmungen « einem Empfindungswissen ein, das sich seiner Struktur nicht bewusst ist, die infinitesimal und - in der unendlichen Komplexität des göttlichen Geis- 
tes - zugleich vollständig ist. Wenn Leibniz sich also zwischen dem unendlich Kleinen und dem unendlich Großen munter hin- und herbewegt, dann verharrt diese Vergleichung letztlich auf der Seite des reinen Denkens und gelangt nicht zu einer Verbindung der Begriffe mit einem Realen. Leibniz macht sich Kant zufolge nicht klar, dass diese Bewegung als Vergleich nur reflexiver Natur ist und hält sie konstitutiv für das, wovon sie spricht.

II. CANGUILHEM: DIE BEGRIFFLICHEN ERFINDUNGEN ENTSCHEIDEN SICH LOKAL, IM DETAIL EINER KRITISCHEN A R B EIT

An Canguilhem interessiert mich in diesem Zusammenhang nicht der Philosoph der Normen, des Normalen und des Pathologischen, nicht der Philosoph, der behauptet, dass Lebenswissen nicht auf physikalische Erkenntnis zu reduzieren sei, und auch nicht der Philosoph, der die Zweckmäßigkeit der mechanistischen Theorien aufzeigt, sondern vielmehr der Philosoph, der die Wissenschaftsgeschichte zu einem philosophischen Unternehmen gemacht hat. Ähnlich wie Alexandre Koyré in seinen Études galiléennes ${ }^{6}$ geht Canguilhem davon aus, dass sich die Möglichkeit zur Erfindung neuer Denkweisen immer dann ergibt, wenn Hindernisse und Schwierigkeiten in neue Probleme umgewandelt werden - was zur Folge hat, dass Begrenzungen und Situierungen von Wissensfeldern an Bedeutung gewinnen. Insofern stimme ich mit Pierre Macherey überein, wenn er daran erinnert, was Canguilhems Denkstil ausmacht. In seinen Kommentaren zu schwer zugänglichen Texten wie etwa d'Alemberts Artikel "Application « in der Encyclopédie, den er Auszügen aus der Science des ingénieurs von Bérnard Belidor gegenüberstellt, führt Canguilhem in der Tat grundlegende Elemente einer Technikphilosophie ein, »die sich auf bestimmte charakteristische Aspekte der Geschichte des Begriffs stützt, der in seinen Wandlungsmomenten erfasst und von dort aus auf seine wesentlichen spekulativen und praktischen Einsatzorte zurückgeführt wird «. ${ }^{7}$ Dass Wissenschaft immer historisch situativ entsteht, ist der Ausgangspunkt von Canguilhems Philosophie.

Die einzige Weise zu begreifen, wie das wissenschaftliche Denken, insbesondere in den Lebenswissenschaften, dazu gelangt, ohne die Dualismen, von denen die Metaphysik lebt, auszukommen, besteht für Canguilhem darin, die Begrenzungen der Wissensfelder in Erscheinung tre- 
ten zu lassen. So benötigt er nicht mehr den Umweg über die kantische Vorstellung, wonach das Phänomen in seiner Begrenzung nur in Bezug auf ein »Jenseits ", ein Ding an sich, einen Sinn annimmt, dessen Leere bewiesen werden muss. Beispielhaft angeführt seien hier die scheinbar "nur historischen « Studien zum Reflexbegriff beginnend mit der ersten Arbeit von 1953 bis zum späteren Artikel über die »Herausbildung des Reflexbegriffs im 19. Jahrhundert « von 1964 oder aber sein berühmter Artikel »Was ist Psychologie? " von $1956 .{ }^{8}$ Wozu dient nun in philosophischer Hinsicht die historische Erkenntnis bei Canguilhem? In den erwähnten Arbeiten zeigt er nicht nur, dass sich der Begriff des Reflexes mindestens ebenso - wenn nicht sogar eher - bei den Vitalisten Willis und Prochaska wie bei den Physiologen herausgebildet hat, die sich in einer retrospektiven, trügerischen Geschichtsauffassung auf Descartes und die Iatromechaniker berufen. Vielmehr zeigt Canguilhem auf gleichsam beiläufige Weise, dass der Reflex insofern von philosophischem Interesse ist, als die Physiologie an seiner Geschichte lernte, sich von den Begriffen »bewusst « und "unbewusst " zu befreien, und zwar dank des Begriffs der sensiblen Fasern, der nicht mehr an den Begriff der Empfindung geknüpft ist. Solange man von Empfindungen spricht, wird man bei der Erforschung der Reflexe zur Annahme verleitet, dass es eine Art unbewusste Empfindung gibt, da sich die auslösende Bewegung der Fasern im Rückenmark und nicht im Gehirn »reflektiert «. Die Entdeckung zweier Nervensysteme, dem zentralen und dem peripheren, scheint so mit der Unterscheidung von willentlichen und unwillentlichen Bewegungen übereinzustimmen, die ihrerseits mit einer philosophisch gefassten Vorstellung von Bewusstsein einhergehen. Doch ist der Wille, die philosophische Unterscheidung zwischen Bewusstem und Unbewusstem in eine strikte Übereinstimmung mit der Physiologie zu bringen, illusorisch. Erst die experimentelle Theorie der neuronalen Erregungsleitung, die sich kreisförmig durch die Fasern ausbreitet, ist unabhängig von der antiken Unterscheidung zwischen Bewusstem und Unbewusstem. Eben darum geht Canguilhems berühmte Kritik an der Psychologie als einer Pseudo-Wissenschaft, die das Bewusstsein zu objektivieren versucht, eng mit seinen wissenschaftsgeschichtlichen Arbeiten über den Reflex parallel. Die sogenannte Wissenschaft von der Psyche konstituiert ihren Gegenstand allein auf Grundlage einer objektivierenden Fehlinterpretation von Descartes. Gerade weil eine Wissenschaft von der Psyche nicht existiert, verkehrte sich die Psychologie historisch so leicht in eine Politik der Verhaltensformen - welche nichts anderes als eine 
Selektion von Individuen zu bestimmten Aufgaben ist, die erfordern, dass Menschen Instrumente und nicht etwa ein epistemischer Wert sind. Die Psyche ist ein nicht-konstituiertes Wissensobjekt. Man kann dies entweder direkt zeigen, indem man die Geschichte der Psychologie aufarbeitet, oder indirekt, indem man nachvollzieht, wie die Physiologie des Reflexes sich, wie oben dargelegt, von dem Begriff der Psyche zu befreien wusste, indem sie zunächst die Metapher der Reflexion der neuronalen Erregungsleitung entlehnte, die sich entlang des zentralen Rückenmarks ausbreitet. Wenn die kopernikanische Revolution der Physiologie der Bewegung in der Trennung der Begriffe des Gehirns und des senso-motorischen Zentrums sowie in der experimentellen Entdeckung exzentrischer Zentren (d. h. des Reflexes) besteht, wird die Konzeption »eines sinnlichen Eindrucks [...], der nicht verspürt wird «, ${ }^{9}$ zur eigentlichen begrifflichen Schwierigkeit. Denn seit Aristoteles ist es nicht der Nerv, der empfindet, sondern die Seele, ob man diese nun als Seele im Rückenmark vermutet oder als Bewusstsein auffasst. Und Canguilhem fügt dem hinzu:

Nach und nach wird jede Referenz auf die subjektive Erfahrung aus der Definition der Sensibilität entfernt oder, genauer, eingeklammert werden. Die Funktion der Sensibilität wird in objektiver Weise durch die histologische Struktur der Erregungsrezeptoren definiert, durch die Richtung der Nervenwirkung in der Nervenfaser. Die Sensibilität wird das zentripetale nervöse System sein. Doch nur, weil es sich nicht länger in den Werken der Physiologie stellt, ist das psychologische Problem der Sensibilität nicht auch schon beseitigt worden. ${ }^{10}$

Diese letzte Bemerkung ist wichtig: Wenn Canguilhem nicht hinzufügen würde, dass die psychologische Frage nach der Natur der Sensibilität bestehen bleibe, könnte seine Position als eine pragmatische verstanden werden. Es würde dann genügen, einen allzu theoretischen Begriff zu beseitigen, um zu einem positiven Wissen Zugang zu erhalten. Dies geschieht beispielsweise in der Psychiatrie mit den Mitteln des DSMKatalogs (»Diagnostic and Statistical Manual of Mental Disorders«), wodurch die Psychopathologie als Theorie in einem beschränkten AntiKonzeptualismus aufgelöst wird. ${ }^{11}$ Da bestimmte Psychopharmaka auf die Effekte dieser oder jener Neurotransmitter einwirken, nimmt man ihre Wirkung als einzige semiologische Leitlinie und erklärt damit jede Theorie als irrelevant, die keinen Bezug aufweist zu den Substanzen, die man mehr oder weniger unter Kontrolle hat. Dies ist keineswegs Can- 
guilhems philosophische Position: Dass die Theorie der neuronalen Erregungsleitung nicht mehr auf das Bewusstsein verweist, stellt für ihn einen Zugewinn an Rationalität dar, eine Weise, den Reflex besser zu verstehen, und nicht, ihn besser zu manipulieren. So sagt Canguilhem noch 1964: "Zu diesem Zeitpunkt [Ende des 19. Jahrhunderts] wird die Seele wieder an die Grenzen der Physiologie geführt, was vielleicht nur bedeutet, dass die Referenz auf die gelebte Erfahrung eingeklammert wurde. ${ }^{12}$ Damit wurde aber noch nicht einer philosophischen Ausarbeitung vorweggegriffen, die sich bezüglich des Begriffs der unbewussten Empfindung, des Bewusstseins und des Unbewussten als notwendig erwies. So weiß man etwa, dass die gesamte Geschichte der Psychoanalyse von diesem Problem durchzogen ist: Hat die klinisch an den Tag gebrachte subjektive Spaltung des Subjekts - in Anbetracht des variablen Abstands, den dieses zu seinen Träumen, zu seinen Symptomen unterhält, oder durch die Kluft, die ihm die Existenz allein erträglich macht - hat diese Spaltung etwas mit der Frage des Bewusstseins zu tun, so wie sie von Descartes gestellt wird? Ist es an dieser Stelle angebracht den Begriff des Unbewussten zu verwenden, um alle Aspekte der subjektiven Spaltung zu vereinen, oder nicht? Dies könnte eine der möglichen Ausrichtungen dieser Frage sein, die dann aber nicht mehr mit derjenigen nach der Seele, auch nicht mit der »empfindenden Seele« bei Aristoteles, zusammenfallen würde. Dies scheint überholt, weil sich die konzeptionellen Fragen anders stellen und nicht etwa weil jegliche Theorie nichtig wäre. Die Epistemologie ist bei Canguilhem eine regionale, weil die Fähigkeit, Grenzen zu bestimmen, über die Begriffe entscheidet, die innerhalb dieser Grenzen keine Gültigkeit mehr haben. Die Probleme, die letztere zu definieren halfen, stellen sich nunmehr in Begriffen, die ihrerseits neu zu definieren sind. Daraus ergibt sich eine wesentliche Konsequenz: So ist es wahrscheinlich, dass es kaum mehr einen Sinn hat, eine Biologie des Bewusstseins betreiben zu wollen, denn die Biologie erschafft ihre Objekte, indem sie gerade aufhört, sich auf Vorstellungen solcher Art zu beziehen.

Worin genau besteht nun die Beziehung zwischen der Begriffserfindung und der Tatsache, dass bis dahin unvermeidliche Begriffe nun aufgeben werden können? Ich habe eingangs behauptet, dass dies gleichzeitig geschieht, ohne dass man die Modalitäten dieser Wechselbeziehung genauer angeben könnte. Sicher ist jedoch, dass das skeptische Moment des Denkens - in dem Sinne, in dem Kant von der skeptischen Methode spricht, die nicht mit Skeptizismus gleichzusetzen ist - es ermöglicht, 
anders zu denken, was ich im Folgenden entlang der Arbeiten von Foucault zeigen werde.

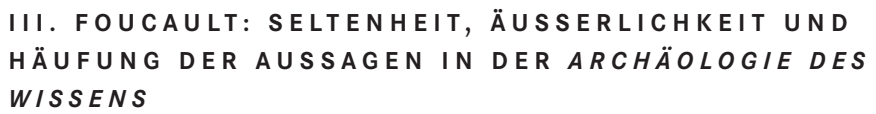

Die Tatsache, dass ein Wissensfeld begrenzt ist, steht bei Foucault in unmittelbarer Beziehung zur internen Kritik, der er seine eigene Arbeit unterzieht: In der Archäologie des Wissens (deren Veröffentlichung 1969, also nur ein Jahr nach den Études d'histoire et de philosophie des sciences von Canguilhem erfolgte) gelangt er dank der Berichtigung von Fehlern und unvermeidlichen Unzulänglichkeiten seiner früheren Bücher zu einer Definition dessen, was er eine Aussage (énoncé) nennt. Wie Foucault gern zu sagen pflegte, begab er sich dabei blind auf unerschlossenes Gebiet, ohne die Methode, die er dabei anwandte, a priori bestimmen zu können. Dies traf für seine Untersuchung der Ausschließungsformen des Wahnsinns zu, des konstitutiven Bruchs, den die Geburt einer medizinischen Klinik markierte, oder aber der Veränderungen der Episteme, was die Herangehensweise an lebende Arten, Sprachen oder Arbeitstätigkeiten angeht. Bei Foucault gibt es weder eine Abhandlung über die Methode, noch eine Kategorientafel oder reine Verstandesprinzipien, und dies aus gutem Grund: Die durch seine diversen Einlassungen in die Geschichte des Wahnsinns, der Medizin oder der sogenannten Humanwissenschaften enthüllten Begriffe werden immer erst nachträglich ausgearbeitet, weil die Methode ihren Gegenständen nicht vorgängig, sondern diesen innewohnend ist. Dabei verhält es sich bei Foucault nicht etwa so, dass sich die eingesetzten Begriffe an die interne Rationalität dessen, wovon sie handeln, anschmiegen. Das Gegenteil ist der Fall, denn noch nie wurde die Vorstellung, wonach die Erkenntnis eines Wirklichkeitsfeldes sich stets von der inneren »Maserung « desjenigen Sachverhalts, dessen Existenz sie verortet, unterscheidet, so weit getrieben wie bei Foucault: Eine Aussage zu verorten, bedeutet demnach, sie in einem Feld der Äußerlichkeit zu platzieren. Doch die intrinsische Beziehung zwischen Kritik und Begrenzung des Felds hängt davon ab, wie die in einem bestimmten Bereich zur Anwendung kommenden Begriffe nachträglich definiert werden, $d$. h. also, nachdem sie zunächst in einem bestimmten Forschungsbereich falsch oder schlecht angewandt wurden. So ist er beispielsweise in Wahnsinn und Gesellschaft fälschli- 
cherweise so vorgegangen, als ob eine einheitliche Erfahrung »des" Wahnsinns die Disparität von Beziehungen der Äußerlichkeit vereinen würde, die dem »Narrenschiff «, der Unvernunft, der Geisteskrankheit ihre jeweilige Konfiguration verleihen. ${ }^{13}$ Es ist zwar unumgänglich mit einem Fehler zu beginnen, doch es ist für einen Denker wichtig, ihn berichtigen zu können, und die Modalitäten dieser Kritik ermöglichen es, die Realität oder Positivität einer diskursiven Praxis zu definieren. Um ein anderes Beispiel zu nennen: Weder die Konstanz der Form noch der Typ der Verkettung zwischen den Propositionen noch der beschreibende Stil machen die Individualität der medizinischen Aussagen im 19. Jahrhundert aus, sondern:

Es schien mir, dass die Medizin sich als eine Folge von deskriptiven Aussagen organisierte. Aber auch hier musste ich diese Anfangshypothese aufgeben und erkennen, dass der klinische Diskurs ebenso eine Gesamtheit von Hypothesen über das Leben und den Tod, von ethischen Entscheidungen, von therapeutischen Entscheidungen, von institutionellen Regelungen, von Unterrichtsmodellen wie eine Gesamtheit von Beschreibungen war. ${ }^{14}$

Foucault räumt letztlich sogar ein, dass er Unrecht gehabt hatte, der Geburt der Klinik den Untertitel Eine Archäologie des ärztlichen Blicks zu geben, ${ }^{15}$ weil dieser glauben mache, zwischen dem Sichtbaren und der medizinischen Sprache bestehe eine einheitliche Beziehung. ${ }^{16}$ Eben diese Richtung hat Deleuze weiterverfolgt: Doch da, wo Foucault von der Aussage spricht, wandelt Deleuze seine Werke ab und liest sie neu als »eine Ontologie des Aussagbaren und Sichtbaren ", was gerade die Verortung der Beziehungen der Äußerlichkeit verunmöglicht, welche eine Aussage von dem Aussagensystem unterscheiden ließe, das Foucault Diskurs nennt. ${ }^{17}$ Eine Aussage wird somit durch die Kritik an dem definiert, womit man sie zunächst verwechselte: die logische Organisation der Propositionen, die grammatikalische und stilistische Struktur des Sinns, die signifikante Struktur eines Textes oder einer Sprache oder auch die Sprechakte, die Vorläufer des »Performativen«. Die Aussage »Träume haben einen Sinn« ist bei Freud nicht dieselbe wie bei Artemidor im 2. Jh. n. Chr. Ein Buch steht vor dem Tod eines Autors nicht für dieselbe Aussage wie nach seinem Tod, da es weder mit Blick auf die Bedingungen intellektuellen Eigentums, noch hinsichtlich der Konfrontation mit anderen Werken in einem vergleichbaren Verhältnis steht.

Eine Begrenzung charakterisiert folglich nicht nur das »nichtdeduktive System « von Verhältnissen, die sich zwischen institutionellen 
Maßnahmen, Wissensformen und Praktiken einstellen, sondern die Bestandteile einer Aussage an sich: »Seltenheit, Äußerlichkeit, Häufung «. Dass eine Aussage selten ist, bedeutet, dass das Regelsystem, das sie für eine bestimmte Zeit realisiert und das eine bestimmte Ebene konstruiert, auf der sich Praktiken, Wissensformen, politische und administrative Maßnahmen kreuzen, individuell ist und dass diese Individualität sehr viel begrenzter ist als beispielsweise die unendliche Anzahl von Sätzen, die die Struktur einer Sprache zulässt. Es ist eben diese Seltenheit, die aus der Aussage ein Gut macht, um das man sich gewissermaßen reißt, was wiederum bestätigt, dass sie aus einem Gemisch von Wissensund Machtformen hervorgeht. Ferner hat ein Diskurs als ein Ensemble von Aussagen auch zeitliche Bedingungen: So bezieht sich das Kriterium der Häufung auf die Weise, in der ein Diskurs einem Modus der Wiederholung, der Remanenz, der Summierbarkeit sowie einem Modus der Rekurrenz unterliegt, die eine bestimmte Form der Dauer einer Aussage in der Zeitdefiniert. Wenn Foucault die Begrenzung oder die Endlichkeit eines neuen Genres definiert - eine Endlichkeit, die sich nicht mehr auf den Gegensatz zu einem Unendlichen bezieht -, dann löst er ebenso wenig wie Canguilhem die Frage, was aus den vorausgehenden Begriffen wird, die infolge einer Kritik aufgegeben wurden. Zuweilen scheint er aus der Begrenzung der Aussagen einfach eine Art Fallstudie zu machen, die mit früheren Sichtweisen vereinbar wäre:

$[\mathrm{M}]$ an erfragt nicht den in einer Epoche den Wörtern 'Melancholie oder ,Wahnsinn ohne Delirium verliehenen Sinn oder den inhaltlichen Gegensatz zwischen `Psychose $<$ und $\gg$ Neurose . Nicht, dass solche Analysen als illegitim oder unmöglich angesehen werden würden, sondern sie sind unpassend, wenn es sich beispielsweise darum handelt zu erfahren, warum die Kriminalität ein Gegenstand der medizinischen Untersuchung geworden ist oder warum die sexuelle Abweichung sich als ein möglicher Gegenstand des psychiatrischen Diskurses abzeichnet. ${ }^{18}$

Manchmal aber führt die Begrenzung der Aussagen sogar zu einer sehr radikalen philosophischen Neuorientierung, da es sich um nichts weniger handelt, als »ohne die Dinge auszukommen«, mithin ohne die Illusion, dass die Wahrheit eines Denkens darin besteht, mit der Architektur dessen übereinzustimmen, von dem es spricht. Die Archäologie ist also das Mittel, durch die Erzeugung begrenzter Objekte ohne eine Ontologie der Sprache auszukommen: 
Die Möglichkeit einer Aussageanalyse muß, wenn sie eingeführt wird, gestatten, die transzendentale Stütze fortzunehmen, die eine bestimmte Form philosophischen Diskurses allen Analysen der Sprache im Namen des Seins dieser Sprache und der Fundierung, wo sie ihren Ursprung nehmen sollte, entgegensetzt. ${ }^{19}$

Der Scharfsinn für »Positivitäten «, der kein Positivismus ist, qualifiziert also ein Denken als ein wesentlich kritisches, was Foucaults spätere Analyse der Beziehungen zwischen Diagnostik über das Aktuelle und Denken bestätigen wird.

Kant hat, um zum Schluss zu kommen, in der transzendentalen Dialektik eine skeptische Methode entwickelt, die auf Gewissheit geht und sich insofern vom Skeptizismus absetzt. Diese kritische Methode steht seither in einer engen Beziehung zur Problematik der Vernunftgrenzen. Ich habe gezeigt, dass Canguilhem und Foucault, die beide an Kant und seine kritische Methode anknüpften, das Problem der Grenzen nicht nur innerhalb der Dialektik der reinen Vernunft zu bestimmen suchten, sondern auf die Frage der Bestimmung empirischer Wissensfelder ausweiteten.

In der Folge wird auch die Problematik der Wahrheit bei Canguilhem und Foucault anders bearbeitet als bei Kant: Ein neuer, skeptischer Begriff der Wahrheit heißt bei Canguilhem immer noch Wahrheit, bei Foucault nur noch »veridicité«. Bei aller Differenz zwischen der »skeptischen Methode " bei Kant und dem skeptischen Moment bei Canguilhem und Foucault stellt jedoch für alle drei Philosophen das skeptische Element des Denkens ein Moment der Erfindung dar und nicht wie für Hegel nur ein Moment der »Ataraxie des Sich-selbst-denkens «, ${ }^{20}$ wie Hegel sich in der Phänomenologie des Geistes ausdrückt.

Aus dem Französischen von Franziska Schottmann und Konrad Petrovszky

\section{A N MERKUNGEN}

1 Monique David-Ménard, La folie de la raison pure. Kant lecteur de Swedenborg (Paris: Vrin, 1990); dies., Les constructions de l'universel. Psychanalyse, philosophie (Paris: PUF, 1997), dt. Konstruktionen des Allgemeinen. Psychoanalyse, Philosophie, übers. v. Hans-Dieter Gondek (Wien: Turia + Kant, 1999).

2 Daher auch der Titel meines zweiten hier zitierten Buchs Konstruktionen des Allgemeinen. 
3 Michel Foucault, Der Gebrauch der Lüste. Sexualität und Wahrheit II, übers. v. Ulrich Raulff u. Walter Seitter (Frankfurt a.M.: Suhrkamp, 1986), S. 15.

4 Siehe Françoise Balibar, »Von Canguilhem zu Haraway « im vorliegenden Band.

5 Immanuel Kant, Kritik der reinen Vernunft, hg. v. Wilhelm Weischedel, 2 Bde. (Frankfurt a.M.: Suhrkamp, 1974), I, S. 306.

6 Alexandre Koyré, Études galiléennes, 3 Bde. (Paris: Hermann, 1939).

7 Pierre Macherey, "Georges Canguilhem: un style de pensée «, in ders., De Canguilhem à Foucault. La force des normes (Paris: La Fabrique, 2009), S. 110-23, hier S. 113.

8 Georges Canguilhem, La formation du concept de réflexe aux XVIIe et XVIIIe siècles (Paris: PUF, 1955), dt. Die Herausbildung des Reflexbegriffs im 17. und 18. Jahrhundert, übers. v. Henning Schmidgen (München: Fink, 2008); ders., "Le concept de réflexe au XIXe siècle", in ders., Études d'histoire et de philosophie des sciences, 7., erw. Aufl. (Paris: Vrin, 1994), S. 295-304; ders., "Qu'est-ce que la psychologie? «, in ders., Études d'histoire, S. 365-81.

9 Canguilhem, Die Herausbildung des Reflexbegriffs, S. 158.

10 Ebd., S. 159-60.

11 Das DSM wird seit 1952 von der American Psychiatric Association herausgegeben und liegt gegenwärtig in revidierter 4. Auflage vor: Diagnostic and Statistical Manual of Mental Disorders: DSM-IV-TR (Washington, DC: American Psychiatric Association, 2000), dt. Henning Saß u. a., Diagnostisches und statistisches Manual psychischer Störungen (DSM-IV-TR) (Göttingen: Hogrefe, 2003).

12 Canguilhem, »Le concept de réflexe au XIXe siècle«, S. 302 (Übersetzung K.P.).

13 Michel Foucault, Wahnsinn und Gesellschaft. Eine Geschichte des Wahns im Zeitalter der Vernunft, übers. v. Ulrich Köppen (Frankfurt a.M.: Suhrkamp, 1973).

14 Michel Foucault, Archäologie des Wissens, übers. v. Ulrich Köppen (Frankfurt a.M.: Suhrkamp, 1981), S. 51.

15 Michel Foucault, Die Geburt der Klinik. Eine Archäologie des ärztlichen Blicks, übers. v. Walter Seitter (München: Hanser, 1973).

16 Foucault, Archäologie des Wissens, S. 92.

17 Ebd., S. 170.

18 Ebd., S. 73.

19 Ebd., S. 165.

20 G.W.F. Hegel, Phänomenologie des Geistes (Hamburg: Meiner, 1952), S. 156. 
Monique David-Ménard, »Die Begrenzung der Wissensfelder bei Kant, Canguilhem und Foucault $\ll$, in Situiertes Wissen und regionale Epistemologie: Zur Aktualität Georges Canguilhems und Donna J. Haraways, hg. v. Astrid Deuber-Mankowsky und Christoph F. E. Holzhey, Cultural Inquiry, 7 (Wien: Turia + Kant, 2013), S. 161-73 <https://doi.org/10.37050/ci-07_08>

\section{REFERENCES}

Canguilhem, Georges, La formation du concept de réflexe aux XVIIe et XVIIIe siècles (Paris: PUF, 1955)

—Die Herausbildung des Reflexbegriffes im 17. und 18. Jahrhundert, übers. u. mit einem Vorwort eingel. v. Henning Schmidgen (München: Fink, 2008) <https://doi.org/10.30965/ 9783846745250>

Études d'histoire et de philosophie des sciences, 7. erw. Aufl. (Paris: Vrin, 1994)

David-Menard, Monique, La folie de la raison pure. Kant lecteur de Swedenborg (Paris: Vrin, 1990)

Les constructions de l'universel. Psychanalyse, philosophie (Paris: PUF, 1997), dt. Konstruktionen des Allgemeinen. Psychoanalyse, Philosophie, übers. v. Hans-Dieter Gondek (Wien: Turia + Kant, 1999)

Foucault, Michel, Archäologie des Wissens, übers. v. Ulrich Köppen (Frankfurt a.M.: Suhrkamp, 1981)

Der Gebrauch der Lüste. Sexualität und Wahrheit II, übers. v. Ulrich Raulff u. Walter Seitter (Frankfurt a.M.: Suhrkamp, 1986)

—Die Geburt der Klinik. Eine Archäologie des ärztlichen Blicks, übers. v. Walter Seitter (München: Hanser, 1973)

— Wahnsinn und Gesellschaft. Eine Geschichte des Wahns im Zeitalter der Vernunft, übers. v. Ulrich Köppen (Frankfurt a.M.: Suhrkamp, 1973)

Hegel, G.W.F., Phänomenologie des Geistes (Hamburg: Meiner, 1952)

Kant, Immanuel, Kritik der reinen Vernunft, hg. v. Wilhelm Weischedel, 2 Bde. (Frankfurt a.M.: Suhrkamp, 1974)

Koyré, Alexandre, Études galiléennes, 3 Bde. (Paris: Hermann, 1939)

Macherey, Pierre, De Canguilhem à Foucault. La force des normes (Paris: La Fabrique, 2009) 